\title{
Características demográficas de la población canina de una ciudad de la Patagonia, Argentina: Implicancias para el manejo de la población canina y la salud humana
}

\section{Demographic characteristics of the canine population of a city in Patagonia, Argentina: Implications for the management of the canine population and human health}

\author{
Gilda Garibotti ${ }^{1}$, Ana Laura Guardamagni ${ }^{2}$, Daniela Zacharías ${ }^{1}$, Gustavo Viozzi ${ }^{3,6}$, \\ Verónica Flores ${ }^{3}$, Basilia Alvarado², Rodrigo Bustamante ${ }^{4}$, Elizabeth Chang Reissig ${ }^{5}$, \\ Tomás González ${ }^{2}$, Carlos Rauque ${ }^{3}$, Karina Santos ${ }^{4}$, Rocío Vega ${ }^{3}$, Melani Walker ${ }^{2}$
}

\section{Resumen}

Se investigaron las características demográficas de la población canina, las cualidades del cuidado de los animales y la ocurrencia de mordeduras o accidentes de tránsito a causa de perros en hogares de San Carlos de Bariloche, en el contexto de un estudio sobre el impacto de la tenencia de perros sobre el medioambiente y la salud de la población. Se realizó una encuesta en una muestra estratificada, polietápica y probabilística. La proporción de hogares con perro fue 69\% y se estimó que había 57082 perros con dueño en la ciudad. El 46\% tenía libre acceso a la vía pública. Entre estos, 57\% no habían sido

\footnotetext{
${ }^{1}$ Departamento de Estadística, Universidad Nacional del Comahue, San Carlos de Bariloche, Argentina

${ }^{2}$ Universidad Nacional del Comahue, San Carlos de Bariloche, Argentina

${ }^{3}$ Laboratorio de Parasitología, Universidad Nacional del Comahue, San Carlos de Bariloche, Argentina

${ }^{4}$ Salud Pública Veterinaria de la Unidad Regional de Salud Ambiental, Ministerio de Salud de la Provincia de Río Negro, San Carlos de Bariloche, Argentina

${ }^{5}$ Instituto de Investigaciones Forestales y Agropecuarias Bariloche, IFAB (INTA-CONICET)

${ }^{6}$ E-mail: gviozzi@gmail.com
}

Recibido: 12 de enero de 2021

Aceptado para publicación: 28 de julio de 2021

Publicado: 27 de octubre de 2021

CLos autores. Este artículo es publicado por la Rev Inv Vet Perú de la Facultad de Medicina Veterinaria, Universidad Nacional Mayor de San Marcos. Este es un artículo de acceso abierto, distribuido bajo los términos de la licencia Creative Commons Atribución 4.0 Internacional (CC BY 4.0) [https:// creativecommons.org/licenses/by/4.0/deed.es] que permite el uso, distribución y reproducción en cualquier medio, siempre que la obra original sea debidamente citada de su fuente original 
vacunados y $90 \%$ no habían sido desparasitados en el último año o lo habían sido menos de cuatro veces. El $31 \%$ de los machos y $77 \%$ de las hembras estaban esterilizados, en tanto que solo $48 \%$ de las perras menores de tres años estaba esterilizada. En 5\% de los hogares, alguno de sus integrantes había sido mordido por un perro y en $19 \%$ se reportó que alguno de los integrantes había sufrido un accidente de tránsito a causa de perros en los últimos cinco años. La falta de supervisión de los perros por parte de sus dueños provee condiciones adecuadas para el aumento no controlado de la población canina, la diseminación de enfermedades zoonóticas, la ocurrencia de mordeduras y accidentes de tránsito.

Palabras clave: demografía de perros con dueño, tenencia de perros, zoonosis, mordeduras, Argentina

\section{Abstract}

The demographic characteristics of the canine population, the traits of animal care and the occurrence of dog bites or traffic accidents caused by dogs in homes in San Carlos de Bariloche were investigated, in the context of a study on the impact of dog ownership on the environment and the health of the population. A survey was conducted in a stratified, multistage, and probabilistic household sample. The proportion of households with dogs was $69 \%$ and it was estimated that there were 57082 owned dogs in the city. The $46 \%$ had free access to public spaces. Among these, $57 \%$ had not been vaccinated and $90 \%$ had not been dewormed in the last year or had been less than four times. Besides, $31 \%$ of the males and $77 \%$ of the females were neutered, while only $48 \%$ of the bitches under three years of age were spayed. In $5 \%$ of the households, one of their members had been bitten by a dog and in $19 \%$ one of the members had suffered a traffic accident caused by dogs in the last five years. The lack of supervision of dogs by their owners provides adequate conditions for the uncontrolled increase in the canine population, the spread of zoonotic diseases, the occurrence of dog bites and traffic accidents.

Key words: demography of dogs with owners, dog ownership, zoonosis, bites, Argentina

\section{INTRODUCCIÓN}

La relación cercana entre los seres humanos y los animales data de muy temprano en la historia de la humanidad. La primera especie en ser domesticada fue el perro, aunque persisten las controversias sobre el momento y lugar de domesticación (Yilmaz, 2017a,b). Desde la antigüedad, los perros le proveyeron al humano protección y compañía, los ayudaron a pastorear y cazar y también fueron utilizados como medio de transporte. Hoy en día, es reconocida la contribu- ción de los perros para prevenir y predecir enfermedades y mejorar el bienestar psicológico de las personas (Wells, 2007; O'Haire, 2010), así como asistentes terapéuticos para el tratamiento de desórdenes de conducta, esquizofrenia, síndrome de Down, autismo y Alzheimer, entre otros (Hawkins et al., 2019, Klimova et al., 2019; Hill et al., 2020).

La convivencia armónica entre la población humana y canina en las ciudades depende de una adecuada supervisión de los perros por parte de sus dueños, lo cual incluye alimentación apropiada, vacunación, 
desparasitación, esterilización y control del desplazamiento. Los perros pobremente supervisados tienen condiciones sanitarias y alimentarias deficientes y son proclives a sufrir accidentes. Tienen también un impacto negativo sobre la salud humana y el medio ambiente, pues los perros vagabundos defecan en la vía pública, diseminan basura y pueden agredir y morder a los transeúntes (Garibotti et al., 2017), condiciones propicias para la dispersión de parásitos zoonóticos, como la equinococosis (OMS, 2019a), toxocariasis (Ma et al., 2018; McManus et al., 2018) y rabia (OMS, 2019b). Además, si no están esterilizados se reproducen sin control, pudiendo generar un serio problema de sobrepoblación canina (Smith et al., 2019). Los perros también pueden representar un problema para la fauna silvestre por depredación, perturbación, hibridación y transmisión de enfermedades (Hughes y Macdonald, 2013).

Relevamientos de la población canina realizados en Argentina y Chile dan cuenta de una alta proporción de hogares con perros, bajos porcentajes de vacunación y altos porcentajes de perros con libre acceso a la vía pública, condiciones propicias para el desarrollo y persistencia de zoonosis (Brusoni et al., 2007; Morales et al., 2009; AcostaJamett et al., 2010; Jornet, 2018; Garibotti et al., 2017). Estudios de heces caninas realizados en San Carlos de Bariloche encontraron huevos de Echinococcus granulosus y de Toxocara sp (Semenas et al., 2014; Flores et al., 2017, 2018), especialmente en barrios de bajos recursos o con características semirurales, en tanto que en las provincias de Neuquén y Chubut se reportaron más de 14 especies de helmintos en heces caninas, muchos de ellos potencialmente patógenos para las personas (Zunino et al., 2000; Sánchez et al., 2003; Torrecillas et al., 2014). Numerosos estudios, por otro lado, revelan la magnitud de la problemática de las mordeduras de perros (Feldman et al., 2004; Zanini et al., 2008; Alfieri et al., 2014; Garibotti et al., 2019) asociada a la transmisión de rabia (Ratsitorahina et al., 2009;
Tenzin et al., 2011; OPS, 2018). En este contexto, el estudio de las características demográficas de la población canina y aspectos de la tenencia de mascotas es esencial para desarrollar e implementar programas de control de la población canina para promover una convivencia armónica y saludable entre el perro y el hombre en las ciudades.

El objetivo de este trabajo fue evaluar las características demográficas de la población canina de San Carlos de Bariloche (Patagonia, Argentina): estimar el tamaño de la población, determinar patrones de tenencia y establecer su relación con indicadores sociales.

\section{Materiales y MéTodos}

El estudio se realizó en San Carlos de Bariloche, ciudad que cuenta con una superficie de $220.3 \mathrm{~km}^{2}$ y en 2010 contaba con 112887 habitantes distribuidos en 35220 hogares (Instituto Nacional de Estadísticas y Censos, 2012). La ciudad está compuesta de un casco urbano con calles asfaltadas, edificios y casas contiguas y una amplia zona con calles de tierra, sin veredas y mayormente casas rodeadas de terreno.

La investigación se basó en una encuesta ad hoc cara a cara sobre tenencia de mascotas realizada en una muestra estratificada, polietápica y probabilística de hogares. Etapas de muestreo: i) selección de radios censales del Censo 2010 siguiendo un muestreo estratificado, y ii) selección sistemática con inicio aleatorio del hogar. Para cada radio, a partir de los datos del Censo 2010, se calculó un índice de nivel socioeconómico como el promedio de años de educación de los habitantes de 25 años o más. Los radios censales fueron estratificados de acuerdo con el índice de nivel socioeconómico. Se definieron tres estratos en base a los terciles del índice: E1: nivel socioeconómico menor, E2: nivel socioeconómico intermedio, y E3: nivel socioeconómico mayor 


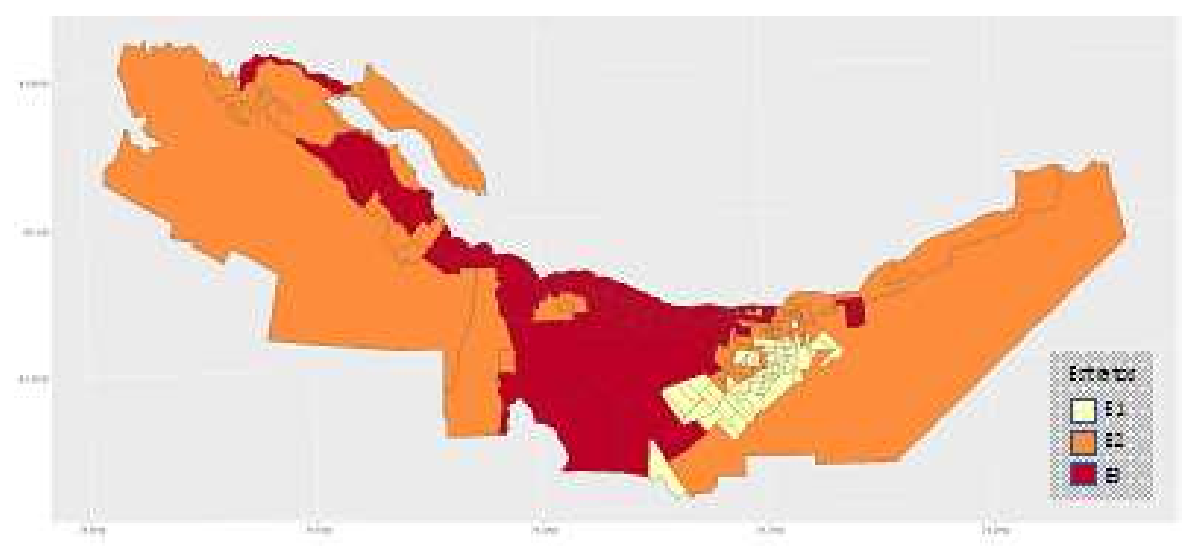

Figura 1. Radios censales de San Carlos de Bariloche con identificación del estrato al que pertenecen. E1: estrato de nivel socioeconómico menor, E2: estrato de nivel socioeconómico intermedio y E3: estrato de nivel socioeconómico mayor

(Figura 1). Se eligieron aleatoriamente cinco radios en cada estrato y se realizó aproximadamente el mismo número de entrevistas por estrato.

Se estimó el porcentaje de hogares con perro, la tasa media de personas por perro, el número medio de animales por hogar y el número total de perros. El número de perros se estimó como la media de perros por hogar (obtenida a partir de la encuesta) multiplicada por el número de hogares de la ciudad, considerando un aumento de $10 \%$ en el número de hogares desde el Censo 2010. Además, se estimó el porcentaje de hogares en los que, en los últimos cinco años, algún miembro había sufrido un accidente de tránsito a causa de perros y el de aquellos en los que alguien había sido mordido por un perro.

Para cada medida, con respecto al perro, se hallaron porcentajes e intervalos de confianza del 95\% (IC95\%). Utilizando la prueba Chi-cuadrado se evaluó la asociación entre esterilización y sexo. Combinando la estimación del número total de perros y el porcentaje de perros con libre acceso a la calle se estimó el número total de perros con dueño que circulan libremente por la calle y a partir de este resultado e información sobre sexo y esterilización, se estimó el número de hembras no esterilizadas con libre acceso a la calle.

Todos los resultados fueron calculados a nivel de la ciudad y por estrato. De acuerdo con el Censo 2010, 39.4\% de los hogares pertenecen a E1, 32.6\% a E2 y $28.0 \%$ a E3. Para obtener los estimadores totales para la ciudad, se tomó una submuestra de encuestas de E2 y E3 de manera de realizar las estimaciones en base a una muestra que tuviese la misma distribución que la población de San Carlos de Bariloche con relación al número de hogares por estrato. El procesamiento y análisis de la información se realizó utilizando el paquete estadístico R 3.2.2 (R Core Team, 2019).

\section{Resultados}

Se entrevistaron 423 hogares, 141 de E1, 142 de E2 y 140 de E3. La submuestra representativa de la ciudad consistió en 357 encuestas (E1: 141, E2: 116, E3:100). El número total de perros fue 576 . 
Cuadro 1. Características de la tenencia de perros en San Carlos de Bariloche, Argentina (2017)

\begin{tabular}{lcccc}
\hline & \multicolumn{3}{c}{ Estrato $^{1}$} & \multirow{2}{*}{ Total } \\
\cline { 2 - 4 } & E1 & E2 & E3 & \\
\hline $\begin{array}{l}\text { Hogares con perro } \\
\text { n (\%) }\end{array}$ & $123(87.2)$ & $93(65.5)$ & $68(48.6)$ & $245(68.6)$ \\
$\begin{array}{l}\text { Relación persona: perro } \\
\begin{array}{l}\text { Media de perros por hogar } \\
\text { (IC 95\%) }\end{array}\end{array}$ & 1.6 & 2.2 & 3.8 & 2.2 \\
\hline
\end{tabular}

${ }^{1}$ Estratos socioeconómicos: E1: menor, E2: intermedio, E3: mayor

\section{Tenencia de Perros}

Se estimó que 68.6\% (IC 95\%: 63.5 a 73.4) de los hogares tenían uno o más perros. Se encontró una mayor proporción de hogares con perros en E1 que en E3 $(\mathrm{p}<0.0001$; Cuadro 1); sin embargo, la tasa de personas por perro fue menor en E1 que en E3. En hogares con perro, $43.3 \%$ tenían un perro, $27.8 \%$ dos perros, $13.5 \%$ tres perros y $15.5 \%$ entre 4 y 10 perros. La proporción de hogares con un único perro fue casi el doble en E3 (66.2\%) que en E1 (34.1\%); la proporción en E2 fue 49.5\%. Siguiendo la misma tendencia, la media de perros por hogar en E1 fue tres veces mayor que en E3 (Cuadro 1).

\section{Sexo, Edad y Manejo de Perros}

En E1 predominaron los machos en tanto que en los otros dos estratos predominaron las hembras, aunque sin diferencias significativas $(\mathrm{p}=0.1448)$. La proporción de perros de menor edad en E1 fue mayor que en E2 y E3 (Cuadro 2).

La proporción de perros esterilizados se asoció positivamente con el nivel socioeconómico del estrato, alcanzando casi el 70\% en E3, a diferencia de E1 donde solo $50.7 \%$ de los perros estaban esterilizados
(Cuadro 2). El 57.4\% de las esterilizaciones habían sido realizadas de manera gratuita, siendo de $77.1 \%$ en E1, $48.3 \%$ en E2 y $22.4 \%$ en E3 $(\mathrm{p}<0.0001)$. Con relación al sexo, $30.8 \%$ de las esterilizaciones se hicieron en machos y $76.7 \%$ en hembras ( $p<0.0001)$. En el caso de los machos, el porcentaje fue similar en todos los estratos ( $\mathrm{p}=0.3161)$, mientras que entre las hembras las diferencias fueron significativas $(\mathrm{p}=0.0451)$ variando entre 72.1 y $88.3 \%$ (Figura 2, izquierda). El $22.7 \%$ de las hembras menores de un año, $61.8 \%$ entre 1 y 3 años, y $91.5 \%$ mayores de 3 años estaban esterilizadas $(\mathrm{p}<0.0001)$. De otra parte, menos de $10 \%$ de las hembras menores de un año estaban esterilizadas en E1 y tanto en E1 como en E2 la proporción de hembras de entre 1 y 3 años esterilizadas fue inferior al 60\% (Figura 2, derecha).

Solamente $55.0 \%$ de los perros habían sido vacunados en los últimos 12 meses, habiendo diferencia significativa entre estratos $(p<0.0001)$. La proporción de perros vacunados en E3 casi duplica la de E1 (Cuadro 2). El porcentaje de perros desparasitado con una frecuencia igual o menor a 4 meses fue de solamente $9.2 \%$, mientras que $75.7 \%$ habían sido desparasitados una única vez o con una frecuencia superior a 4 meses y $15.1 \%$ nunca habían sido desparasitados, habiendo diferencias significativas entre estratos; sin 
Cuadro 2. Distribución por sexo y edad y descripción del cuidado de los perros en San Carlos de Bariloche, Argentina (2017)

\begin{tabular}{|c|c|c|c|c|c|c|c|c|c|}
\hline & \multirow{2}{*}{\multicolumn{2}{|c|}{ Total }} & \multicolumn{6}{|c|}{ Estrato $^{1}$} & \multirow{3}{*}{ p-valor } \\
\hline & & & \multicolumn{2}{|c|}{ E1 } & \multicolumn{2}{|c|}{ E2 } & \multicolumn{2}{|c|}{ E3 } & \\
\hline & $\mathrm{n}$ & $\%$ & $\mathrm{n}$ & $\%$ & $\mathrm{n}$ & $\%$ & $\mathrm{n}$ & $\%$ & \\
\hline Sexo masculino & 252 & 48.6 & 156 & 52.0 & 85 & 49.1 & 42 & 40.8 & 0.1448 \\
\hline Edad (años) & & & & & & & & & $<0.0001$ \\
\hline$<1$ & 58 & 13.3 & 42 & 19.0 & 15 & 8.7 & 7 & 6.9 & \\
\hline $1-3$ & 147 & 33.6 & 88 & 39.8 & 53 & 30.8 & 27 & 26.5 & \\
\hline$>3$ & 232 & 53.1 & 91 & 41.2 & 104 & 60.5 & 68 & 66.7 & \\
\hline Esterilizado & 275 & 54.1 & 149 & 50.7 & 91 & 53.5 & 71 & 69.6 & 0.0038 \\
\hline Vacunado & 275 & 55.0 & 128 & 44.4 & 99 & 59.3 & 83 & 84.7 & $<0.0001$ \\
\hline Desparasitado & & & & & & & & & 0.0060 \\
\hline Nunca & 67 & 15.1 & 44 & 16.6 & 14 & 10.8 & 11 & 12.1 & \\
\hline Cada 4 m o más & 336 & 75.7 & 206 & 77.7 & 94 & 72.3 & 70 & 76.9 & \\
\hline $\begin{array}{l}\text { Cada } 4 \text { m o } \\
\text { menos }\end{array}$ & 41 & 9.2 & 15 & 5.7 & 22 & 16.9 & 10 & 11.0 & \\
\hline Libre acceso a la calle & 238 & 46.0 & 166 & 55.3 & 62 & 36.0 & 30 & 29.1 & $<0.0001$ \\
\hline
\end{tabular}

${ }^{1}$ Estratos socioeconómicos: E1: menor, E2: intermedio, E3: mayor

embargo el porcentaje de perros desparasitados con frecuencia menor o igual a 4 meses fue baja en todos ellos, no superando 20\% (Cuadro 2). Por último, la proporción de perros con libre acceso a la vía pública fue $46 \%$, siendo mayor en E1 que en E2 y E3 $(\mathrm{p}<0.0001$; Cuadro 2).

\section{Población Canina}

El número estimado de perros con dueño en San Carlos de Bariloche fue 57082 y casi 26000 tenían libre acceso a la vía pública (Cuadro 3). El número estimado de hembras no esterilizadas fue 6550 , de las cuales casi 2800 tenían libre acceso a la vía pública (Cuadro 3).

\section{Perros y Salud Humana}

Alguno de los integrantes de los hogares $(4.7 \%)$ había sido mordido por un perro en los últimos 5 años, sin diferencias signifi- cativas entre estratos. En $56.1 \%$ de los casos se tuvo que recurrir al médico (E1: 67.9\%, E2: $42.9 \%, E 3: 39.3 \%, p=0.0142)$, en $17.9 \%$ de los casos se realizó la denuncia y en 7.5\% se realizó el control antirrábico al perro. En $99.0 \%$ de los casos el perro que mordió no era propio, y sin diferencias significativas entre estratos.

En 19.4\% de los hogares se reportó que alguno de sus integrantes había sufrido un accidente de tránsito a causa de perros en los últimos 5 años (E1 25.7\%, E2 17.7\%, E3 $12.1 \%)(\mathrm{p}=0.0134)$.

\section{Discusión}

En Argentina la problemática de la población canina poco supervisada se ha puesto en evidencia en algunos relevamientos esporádicos, pero no existen diagnósticos de situación actualizados en forma periódica que 

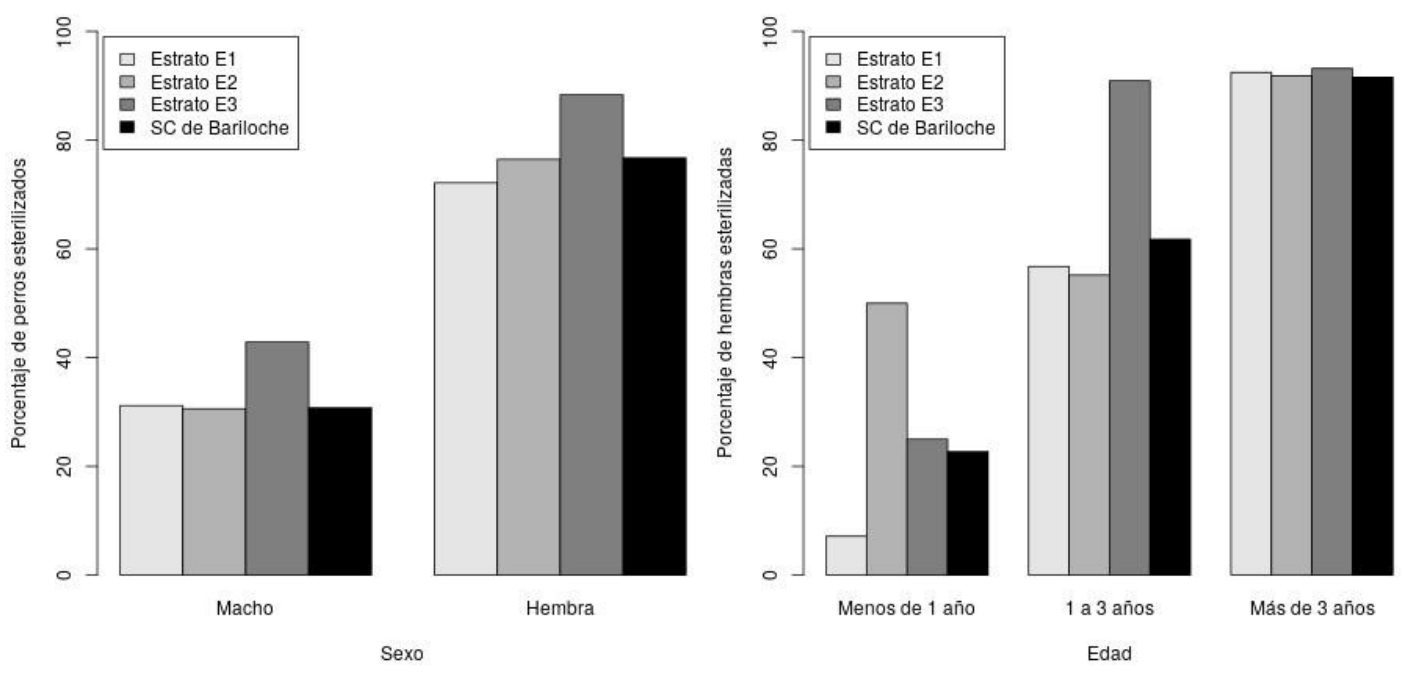

Figura 2. Población canina esterilizada en San Carlos de Bariloche, Argentina (2017), según sexo (izquierda) y grupo etario (derecha) distribuido por estrato socioeconómico (E1: estrato de menor nivel, E2: estrato de nivel intermedio; E3: estrato de mayor nivel)

Cuadro 3. Magnitud de la población canina (número estimado \pm error estándar) con dueño en San Carlos de Bariloche, Argentina (2017)

\begin{tabular}{|c|c|c|c|c|}
\hline & \multirow{2}{*}{ Total } & \multicolumn{3}{|c|}{ Estrato $^{1}$} \\
\hline & & E1 & E2 & E3 \\
\hline Total de perros & $57,082 \pm 3,200$ & $33,395 \pm 2,291$ & $15,379 \pm 1,330$ & $7,968 \pm 889$ \\
\hline $\begin{array}{l}\text { Perros con libre acceso a } \\
\text { la vía pública }\end{array}$ & $25,901 \pm 2,319$ & $17,998 \pm 1,886$ & $5,551 \pm 837$ & $2,321 \pm 436$ \\
\hline Hembras no esterilizadas & $6,550 \pm 950$ & $4,352 \pm 775$ & $1,726 \pm 412$ & $545 \pm 202$ \\
\hline $\begin{array}{l}\text { Hembras no esterilizadas } \\
\text { con libre acceso a la vía } \\
\text { pública }\end{array}$ & $2,783 \pm 680$ & $2,232 \pm 624$ & $366 \pm 181$ & $156 \pm 110$ \\
\hline
\end{tabular}

${ }^{1}$ Estratos socioeconómicos: E1: menor, E2: intermedio, E3: mayor

den cuenta del tamaño y características demográficas de la población, su evolución temporal y asociación con indicadores sociales.

La proporción de hogares con perro $(68.6 \%)$ fue similar a la reportada en otros estudios del país. Así, Brusoni et al. (2007) encontraron que $57 \%$ de los hogares de San
Martín de los Andes tenían perros, mientras que Jornet (2018) reportaron $80.6 \%$ de hogares de Neuquén con perros. El porcentaje de hogares con perro en zonas urbanas de Chile fue entre 49 y $57 \%$ (Morales et al., 2009; Acosta-Jamett et al., 2010) mientras que en Brasil fue entre 55 y $65 \%$ (Martins et al., 2013; Baquero et al., 2015; Trapp et al., 
2015). Esto contrasta con el $25 \%$ de hogares con perro en países como el Reino Unido (Pet Food Manufacturers Association, 2019) y de $38.4 \%$ en Estados Unidos (American Veterinary Medical Association, 2018).

La media de perros por hogar varió significativamente entre estratos, siendo de 2.2 en el estrato de menor nivel socioeconómico y 0.7 en el de mayor nivel socioeconómico. Brusoni et al. (2007) hallaron una media de 0.62 perros por hogar en San Martín de los Andes, media muy inferior a la hallada en este estudio (1.5 perros/hogar), pero al igual que en este estudio, encontraron una tendencia a mayor número medio en sectores con terrenos de menor valor. Jornet (2018) también halló diferencias en la media de perros por hogar de acuerdo con los barrios de la ciudad de Neuquén, con valores entre 0.6 y 2.4 perros por hogar, similar a lo hallado en este estudio. De otra parte, Morales et al. (2009) en Viña del Mar, Chile, encontraron entre 0.71 y 1.27 perros por vivienda según el sector de la ciudad, mientras que Martins et al. (2013) en San Pablo, Brasil, no hallaron asociación entre los ingresos del hogar y el número de perros.

Este estudio muestra una baja proporción de perros esterilizados, siendo mayor en hembras (77\%) que en machos (31\%) y con grandes diferencias entre estratos (E1: $72 \%$, E2: 76\%, E3: 88\%). Brusoni et al. (2007) reportaron una tasa similar de hembras esterilizadas, pero solo de $2.8 \%$ de machos castrados; asimismo, al igual que en este estudio, el porcentaje de hembras esterilizadas en los estratos de menor nivel socioeconómico tendía a ser menor que en los estratos de mayor nivel socioeconómico. Estudios en Brasil y Chile encontraron entre 2 y $23 \%$ de perros esterilizados, cifras muy inferiores a los hallados en el presente estudio (Molina Loyola, 2005; Acosta-Jamett et al., 2010; Baquero et al., 2015; Trapp et al., 2015).

La tercera parte de los entrevistados que no esterilizaron a alguno de sus perros no dieron una razón. Por otro lado, entre los que dieron razones para no esterilizar, 19\% manifestó que quería que tuviese cría, 16\% que el perro era muy joven, $15 \%$ que no tiene facilidad para llevarlo a esterilizar y $14 \%$ que no es necesario porque nunca está suelto o es muy mayor. Jornet (2018) encontró que $50 \%$ de las personas que no habían esterilizado a sus perros manifestaban no tener deseos de hacerlo o no estar decididos. En este sentido, el éxito de las campañas de esterilización puede verse comprometido por la opinión de la población al respecto. Morales et al. (2009) encontraron que la tasa de fecundidad específica estimada por edad es mayor en perros de entre 1 y 4 años, de allí que es preocupante observar que $52 \%$ de las hembras menores de 3 años no se encontraban esterilizadas.

Casi la mitad de los perros accedían a la vía pública sin supervisión. Brusoni et al. (2007) encontraron una proporción similar, especialmente en sectores de menores recursos. Morales et al. (2009) en Viña del Mar, Chile, registraron una tasa de confinamiento permanente superior al $75 \%$ indicando un mayor compromiso respecto del cuidado de los perros. Entre los perros con libertad para deambular, 57\% no habían sido vacunados y $90 \%$ no habían sido desparasitados en los últimos 12 meses o lo habían sido con una frecuencia mayor a cuatro meses; condiciones propicias para la diseminación de enfermedades zoonóticas, incluyendo las parasitarias (Alonso et al., 2001; Milano y Oscherov, 2005; Semenas et al., 2014; Torrecillas et al., 2014; Flores et al., 2017, 2018). Es importante destacar el estudio de seroprevalencia de Toxocara canis en la ciudad de La Plata de Radman et al. (2000), donde se encontró $39 \%$ de casos positivos en personas, con una prevalencia mayor en menores de 15 años. Asimismo, se reporta una asociación significativa entre parásitos intestinales en humanos con presencia de perros en hogares (Gamboa et al., 2009; Vargas et al., 2016).

Los perros en la vía pública pueden morder, causar accidentes de tránsito e intimidar a peatones, ciclistas y motociclistas. El 
estudio estimó que en la ciudad hay 25900 perros con dueño con acceso no supervisado a la vía pública. Las lesiones por mordeduras de perro pueden tener consecuencias físicas y psicológicas muy importantes (Pisapía et al., 2008; Bergagna, 2009). Cerca de 5\% de los hogares entrevistados tenía un integrante que había sido mordido por un perro en los últimos 12 meses, teniendo que recurrir al médico en $56 \%$ de los casos. Jornet (2018) encontró un porcentaje muy superior, donde alguien había sido mordido por un perro en $23 \%$ de las viviendas. Numerosos estudios encontraron que los más afectados por mordeduras de perros son los niños (Zanini et al., 2008; Alfieri et al., 2014; OMS, 2018).

El conocimiento sobre los riesgos que representan los perros sobre la salud humana y las medidas de prevención es la base para establecer el compromiso de la población con relación a la tenencia responsable. Garibotti et al. (2020) en una propuesta de enseñanza para la escuela secundaria mediante el abordaje de problemas comunitarios desarrollaron una intervención en numerosas escuelas de la ciudad en la que se abordó la tenencia responsable de mascotas. Asimismo, Alvarado (2020) desarrolló actividades de promoción de la salud sobre lesiones por mordeduras de perro utilizando Facebook, material impreso y videos. Es fundamental desarrollar programas para el control de la población canina basados en la tenencia responsable, priorizando la salud pública, donde se incluyan estrategias de educación, sistemas de identificación que permitan responsabilizar legalmente a los dueños, campañas de esterilización, captura de animales sueltos y sistema de adopción.

\section{Conclusiones}

- Se estimó que en San Carlos de Bariloche hay cerca de 26000 perros con dueño con libre acceso a la vía pública. Entre éstos, 57\% no habían sido vacunados y $90 \%$ no habían sido desparasitados en el último año o lo habían sido menos de cuatro veces.
- $\quad$ El $22.7 \%$ de las hembras menores de 1 año, $61.8 \%$ de las de entre 1 y 3 años y $91.5 \%$ de las mayores de 3 años estaban esterilizadas.

- En 5\% de los hogares alguno de sus integrantes había sido mordido por un perro y en $19 \%$ se reportó que alguno de los integrantes había sufrido un accidente de tránsito a causa de perros en los últimos cinco años.

\section{Agradecimientos}

La investigación fue parcialmente financiada por la Universidad Nacional del Comahue, subsidio de la Secretaría de Investigación B223 y por la Agencia Fondo para la Investigación Científica y Tecnológica (FONCYT) PICT 1385-2017.

\section{Literatura Citada}

1. Acosta-Jamett G, Cleaveland $S$, Cunningham A, Bronsvoort B. 2010.

Demography of domestic dogs in rural and urban areas of the Coquimbo region of Chile and implications for disease transmission. Prev Vet Med 94: 272-281. doi: 10.1016/j.prevetmed.2010.01.002

2. Alfieri A, Apa M, Faini M, Frati D. 2014. Animal's attacks: epidemiological situation in Rosario city. Year 2012. UNR Journal 1: 1945-1949.

3. Alonso J, Stein M, Chamorro M, Bojanich M. 2001. Contamination of soils with eggs of Toxocara in a subtropical city in Argentina. J Helminthol 75: 165-168.

4. Alvarado B. 2020. Lesiones por mordeduras de perro en la ciudad de San Carlos de Bariloche: Características y estrategias de prevención. Tesis de Licenciatura en Enfermería. Argentina: Univ. Nacional de Comahue. 54 p.

5. American Veterinary Medical Association. 2018. U.S. pet ownership statistics. [Internet]. Disponible en: https:/ /www.avma.org/resources-tools/reportsstatistics/us-pet-ownership-statistics 
6. Baquero O, Chiozzotto E, García R, Amaku M, Ferreira F. 2015. Demographic characteristics of owned dogs and cats of Votorantim, São Paulo State, Brazil. Cienc Rural 45: 2039-2043. doi: 10.1590/0103-8478cr20141646

7. Bergagna H. 2009. Municipios no eutanásicos: perros y zoonosis. Desde la Patagonia 6: 20-24.

8. Brusoni C, Dezzotti A, Fernandez J, Lara J. 2007. Tamaño y estructura de la población canina en San Martín de los Andes (Neuquén). Anal Vet 27: 11-23.

9. Feldman K, Trent R, Jay M. 2004. Epidemiology of hospitalizations resulting from dog bites in California, 1991-1998. Am J Public Health 94: 1940-1941. doi: 10.2105/ajph.94.11.1940

10. Flores V, Viozzi G, Garibotti G, Zacharías D. 2017. Echinococcosis and other parasitic infection in domestic dogs from urban areas of an Argentinean Patagonian city. Medicina (B Aires) 77: 469-74.

11. Flores V, Viozzi G, Garibotti G, Zacharías D. 2018. Parásitos en perros urbanos de una ciudad patagónica en la Argentina. Salud i Ciencia 23: 286-289.

12. Gamboa M, Navone G, Kozubsky L, Costas M, Cardozo M, Magistrello P. 2009. Protozoos intestinales en un asentamiento precario: manifestaciones clínicas y ambiente. Acta Bioquim Clin L 43:213-218.

13. Garibotti G, Zacharías D, Alvarado B. 2019. Perros en la ciudad: un problema peludo. Desde la Patagonia 16: 16-19.

14. Garibotti G, Zacharías D, Flores V, Catriman S, Falconaro A, Kabaradjian S, Luque ML, et al. 2017. Tenencia responsable de perros y salud humana en barrios de San Carlos de Bariloche. Medicina (B Aires) 77: 309-313.

15. Garibotti G, Zacharías D, Treuque J, Guardamagni A, Huaylla C, Vega R, Miori G, et al. 2020. Una propuesta de enseñanza de estadística en la escuela secundaria mediante el abordaje de problemas comunitarios. Contextos de Educación 28: 1-14.
16. Hawkins E, Hawkins R, Dennis M, Williams J, Lawrie S. 2019. Animalassisted therapy for schizophrenia and related disorders: A systematic review. J Psychiatr Res 115: 51-60. doi: 10.1016/ j.jpsychires.2019.05.013

17. Hill J, Ziviani J, Driscoll C, Teoh AL, Chua JM, Cawdell-Smith J. 2020. Canine assisted occupational therapy for children on the autism spectrum: a pilot randomised control trial. J Autism Dev Disord 50: 4106-4120. doi: 10.1007/ s10803-020-04483-7

18. Hughes J, Macdonald D. 2013. A review of the interaction between freeroaming domestic dogs and wildlife. Biol Conserv 157: 341-351. doi: 10.1016/j.biocon.2012.07.005

19. Instituto Nacional de Estadísticas y Censos. 2012. Mapas temáticos Censo 2010 (GEOCENSO). [Internet]. Disponible en: https://sig.indec.gov.ar/censo2010/

20. Jornet J. 2018. Elaboración de un plan de negocios que estudia la viabilidad de instalar un centro veterinario 24 horas en el límite de las Ciudades de Plaza Huincul y Cutral Có en la Provincia de Neuquén. Tesis de Licenciatura en Administración. Chile: Univ. Nacional del Comahue. 164p

21. Klimova B, Toman J, Kuca K. 2019. Effectiveness of the dog therapy for patients with dementia - a systematic review. BMC Psychiatry 19: 276. doi: 10.1186/s12888-019-2245-x

22. Ma G, Holland CV, Wang T, Hofmann A, Fan C, Maizels RM, Hotez PJ, et al. 2018. Human toxocariasis. Lancet Infect Dis 18: e14-e24. doi: 10.1016/ S1473-3099(17)30331-6

23. Martins CM, Mohamed A, Guimarães AM, de Barros Cda C, Pampuch Rdos $S$, Svoboda W, Garcia Rde C, et al. 2013. Impact of demographic characteristics in pet ownership: modeling animal count according to owners income and age. Prev Vet Med 109: 213-218. doi: 10.1016/ j.prevetmed.2012.10.006 
24. McManus R, Hamilton C, Holland C. 2018. Toxocara spp. In Rose JB, Jiménez-Cisneros B (eds). Global water pathogen project. [Internet]. Available in: www.waterpathogens.org/book/ toxocara

25. Milano A, Oscherov E. 2005. Contaminación de aceras con enteroparasitos caninos en Corrientes, Argentina. Parasitol Latinoam 60: 82-5.

26. Molina Loyola C. 2005. Estudio sobre tenencia responsable de mascotas caninas en la ciudad de Rancagua. Tesis de Médico Veterinario. Chile: Univ. Iberoamericana de Ciencias y Tecnología. 19 p.

27. Morales M, Varas C, Ibarra L. 2009. Caracterización demográfica de la población de perros de Viña del Mar, Chile. Arch Med Vet 41: 89-95. doi: 10.4067/ S0301-732X200900010001

28. O'Haire M. 2010. Companion animals and human health: benefits, challenges, and the road ahead. J Vet Behav 5: 226234. soi: 10.1016/j.jveb.2010.02.002

29. [OMS] Organización Mundial de la Salud. 2018. Mordeduras de animales. [Internet]. Disponible en: www.who.int/ es/news-room/fact-sheets/detail/animalbites

30. [OMS] Organización Mundial de la Salud. 2019a. Equinococosis. [Internet]. Disponible en: www.who.int/ es/news-room/fact-sheet/detail/ eccinococcosis

31. [OMS] Organización Mundial de la Salud. 2019b. Rabia. [Internet]. Disponible en: www.who.int/es/news-room/ fact-sheets/detail/rabies

32. [OPS] Organización Panamericana de la Salud. 2018. Cuatro países de América Latina y el Caribe, los únicos donde la rabia canina sigue actualmente viva. [Internet]. Disponible en: www.paho.-org/hq/index.php?option=com content\&view $=$ article\&id $=$. 14665:rabies-per-sists-in-only-fourcountries-of-latin-america-and-the caribbean\&Itemid $=-135 \&$ lang $=$ es
33. Pet Food Manufacturers Association. 2019. Pet population 2019. [Internet]. Available in: https://www.pfma.org.uk/ pet-population-2019

34. Pisapía G, Molina J, Marcos E. 2008. Epidemiología de las lesiones por mordeduras de perro. Medicina (B Aires) $68: 184-185$.

35. $R$ Core Team. 2019. R: A language and environment for statistical computing. $R$ foundation for statistical computing. [Internet]. Available in: www.Rproject.org

36. $R$ Radman N, Archelli S, Fonrouge R, Guardis M, Linzitto O. 2000. Human toxocariosis. Its seroprevalence in the city of La Plata. Mem I Oswaldo Cruz 95: 281-285. doi: 10.1590/s007402762000000300001

37. Ratsitorahina M, Rasambainarivo J, Raharimanana S, Rakotonandrasana H, Andriamiarisoa M, Rakalomanana F, Richard $\boldsymbol{V}$. 2009. Dog ecology and demography in Antananarivo, 2007. BMC Vet res 5: 21. doi: 10.1186/17466148-5-21

38. Sánchez P, Raso S, Torrecillas $C, M e$ llado I, Nancufil A, Oyarzo C. Flores $M$, et al. 2003. Contaminación biológica con heces caninas y parásitos intestinales en espacios públicos urbanos en dos ciudades de la Provincia del Chubut. Patagonia Argentina. Parasitol Latinoam 58: 131-135. doi: 10.4067/S071777122003000300008

39. Semenas L, Flores V, Viozzi G, Vazquez G, Perez A, Ritossa L. 2014. Helmintos zoonóticos en heces caninas de barrios de Bariloche (Río Negro, Patagonia, Argentina). Rev Argent Parasitol 2: 22-27.

40. Smith LM, Hartmann S, Munteanu AM, Dalla Villa P, Quinnell RJ, Collins LM. 2019. The effectiveness of dog population management: a systematic review. Animals 9: 1020 doi: 10.3390/ ani9121020 
41. Tenzin, Dhand NK, Gyeltshen T, Firestone S, Zangmo C, Dema C, Gyeltshen R, Ward MP. 2011. Dog bites in humans and estimating human rabies mortality in rabies endemic areas of Bhutan. PLoS Negl Trop Dis 5: e1391. doi: 10.1371/journal.pntd.0001391

42. Torrecillas C, Mellado I, Resser C, Becquer S, Sánchez Thevenet P, Catalá C, et al. 2014. Parásitos de interés zoonótico y parasitosis intestinales humanas: situación y gestión de soluciones a escala local en una ciudad de Patagonia (Comodoro Rivadavia, Chubut, Argentina). Rev Argent Zoonosis Enf Infec Emerg 9: 35-37.

43. Trapp S, Maeda M, Kemper B, Barca Junior F, Freire $R$, PrettoGiordano L, Selwyn H. 2015. Population demographic survey and ownership of pet dogs and cats from a small city of southern Brazil. Semin-Cienc Agrar 36: 3211-3226.

44. Vargas C, Torres P, Jerci M, Lobos M, Oyarce A, Miranda J, Ayala S. 2016. Frequency of anti-Toxocara spp antibodies in individuals attended by the Centro de Salud Familiar and environ- mental contamination with Toxocara canis eggs in dog feces, on the coastal Niebla town, Chile. Rev Inst Med Trop Sao Paulo 58: 62. doi: 10.1590/S16789946201658062

45. Wells D. 2007. Domestic dogs and human health: an overview. Br J Health Psychol 12: 145-56. doi: 10.1348/135910706X103284

46. Yilmaz O. 2017a. Controversies of origin of domestic dog - I - Reference of evolution factors of wolf. IOSR J Agric Vet Sci 4: 472-475. doi: 10.21276/ sjavs.2017.4.11.6

47. Yilmaz, O. 2017b. Controversies of origin of domestic dog - II - References of fossil dogs. IOSR J Agric Vet Sci 4: 476-483. doi: 10.21276/sjavs.2017.4.11.7

48. Zanini F, Padinger P, Elissondo $M$, Pérez H. 2008. Epidemiología de las lesiones por mordedura de perro en Tierra del Fuego, Argentina. Medicina 68: 1-5.

49. Zunino M, De Francesco M, Kuruc J, Schweigmann N, Wisnivesky-Colli M, Jensen O. 2000. Contaminación por helmintos en espacios públicos de la provincia de Chubut, Argentina. Bol Chil Parasitol 55: 3-4. 